\title{
EMBEDDING PRODUCTS IN SYMMETRIC PRODUCTS OF CONTINUA
}

\author{
By \\ Enrique Castañeda-Alvarado and Javier Sánchez-Martínez
}

\begin{abstract}
Let $X$ be a continuum. For each natural number $n, F_{n}(X)$ is the $n^{\text {th }}$-symmetric product of $X$ and $X^{n}$ is the product of $X$ with itself $n$ times. In this paper we consider the problem of determining the continua $X$ such that $X^{n}$ can be embedded in $F_{n}(X)$. Moreover, we characterize finite graphs $X$ for which $X^{2}$ is embeddable in $F_{2}(X)$.
\end{abstract}

\section{Introduction}

The symbol $\mathbf{N}$ will denote the set of positive integers. A continuum means a compact connected and metric space. Given a continuum $X$ and $n \in \mathbf{N}$, we denote by $X^{n}$ the product of $X$ with itself $n$ times with the product topology and by $F_{n}(X)$ the hyperspace of all nonempty subsets of $X$ with at most $n$ points, equipped with the Hausdorff metric (see [13, Definition 0.1, p. 1]). This is the so called $n^{\text {th }}$-symmetric product of $X$. It is known that $F_{n}(X)$ is a continuous image of $X^{n}$ (see [2, p. 877]). Symmetric products were introduced by K. Borsuk and S. Ulam in [2]. They proved that, for $I=[0,1]$ and $n=1,2,3, F_{n}(I)$ is homeomorphic to $I^{n}$; for $n \geq 4, F_{n}(I)$ is not homeomorphic to any subset of $\mathbf{R}^{n}$ and $F_{2}\left(S^{1}\right)$ is homeomorphic to Möbius strip, where $S^{1}$ is a simple closed curve. In [11], R. Molski proved that $F_{2}\left(I^{2}\right)$ is homeomorphic to the 4-cell and for $n \geq 3$ neither $F_{n}\left(I^{2}\right)$ nor $F_{2}\left(I^{n}\right)$ is homeomorphic to any subset of $\mathbf{R}^{2 n}$. In [3], R. Bott corrected Borsuk's [1] statement that $F_{3}\left(S^{1}\right)$ is homeomorphic to $S^{1} \times S^{2}$ by showing that $F_{3}\left(S^{1}\right)$ is homeomorphic to the 3 -sphere $S^{3}$. Since $S^{1} \times S^{1}$ can not be embedded in $F_{2}\left(S^{1}\right)$, a natural problem arises to determine continua $X$ such

2000 Mathematics Subject Classification: Primary 54B10, 54B20, 54C25, 54F15.

Key words and phrases: Continuum, product, symmetric products, self-homeomorphic, embedding.

The second author was supported in part by CONACyT.

Received September 10, 2014.

Revised May 20, 2015. 
that $X^{n}$ can be embedded in $F_{n}(X)$. The paper consists of four section. In Section 2, we give necessary definitions. In Section 3, we characterize finite graphs $X$ for which $X^{2}$ is embeddable in $F_{2}(X)$. Finally, in Section 4, we study some continua $X$ for which $X^{n}$ can be embedded in $F_{n}(X)$.

\section{Definitions}

Given a continuum $Z$ and a subset $A$ of $Z, \operatorname{cl}_{Z}(A), \operatorname{int}_{Z}(A)$ and $\partial_{Z}(A)$ denote, respectively, the closure, interior and boundary of $A$ in $Z$. The symbol $|A|$ denotes the cardinality of $A$.

By a graph we mean a continuum which can be written as the union of finitely many arcs any two of which are either disjoint or intersect only in one or both of their end points. By an edge of a graph $X$ we shall always mean one of those arcs. The end points of the edges of $X$ are called vertices of $X$. Given a point $x \in X$ and $n \in \mathbf{N}$, the order of $X$ at $x$, denoted by $\operatorname{ord}(x, X)$, is $n$ provided that for every $\varepsilon>0$ there exists an open set $U$ of $X$ containing $x$ such that $\operatorname{diam}(U)<\varepsilon$ and $\partial_{X}(U)$ consists of exactly $n$ points (see [14, Lemma 9.7, p. 143]). For each vertex $v \in X$ we have either $\operatorname{ord}(v, X)=1$ if $v$ is an end point of $X$ or $\operatorname{ord}(v, X) \geq 2$ otherwise. If $\operatorname{ord}(v, X) \geq 3$, then $v$ is called a ramification point of $X$. The set of all ramification points of $X$ will be denoted by $R(X)$. By a simple $n-o d(n \geq 3)$ we mean a graph $X$ with only one ramification point, exactly $n$ end points and without circles. A simple $3-o d$ will be called a simple triod. A complete graph $K_{m}$ is a graph with exactly $m$ vertices such that any two vertices are joined by an edge of the graph. Let $V$ be the set of vertices of graph $G ; G$ is a bipartite graph with vertex clases $V_{1}$ and $V_{2}$ if $V=V_{1} \cup V_{2}$, $V_{1} \cap V_{2}=\varnothing$ and each edge of $G$ joins a vertex of $V_{1}$ to a vertex of $V_{2}$. The graph $G$ is said bipartite complete if each vertex of $V_{1}$ is joined to every vertex of $V_{2}$ by edges of $G$, if $\left|V_{1}\right|=m$ and $\left|V_{2}\right|=n ; G$ is denoted by $K_{m, n}$. Given a continuum $X$, the cone over $X$ is the quotient space $X \times[0,1] / X \times\{1\}$ (see [9, Definition 5.1, p. 126]). The cone over $X$ will be denoted by cone $(X)$.

Given open subsets $U_{1}, \ldots, U_{m}$ of a continuum $X$, let

$\left\langle U_{1}, \ldots, U_{m}\right\rangle=\left\{A \in F_{n}(X): A \subset \bigcup_{i=1}^{m} U_{i}\right.$ and for each $\left.i \in\{1, \ldots, m\}, A \cap U_{i} \neq \varnothing\right\}$.

It is known that the sets of the form $\left\langle U_{1}, \ldots, U_{m}\right\rangle$ form a basis for the topology of $F_{n}(X)$ called the Vietoris topology (see [13, Theorem 0.11, p. 9]), and that the Vietoris topology and the topology induced by the Hausdorff metric are the same (see [13, Theorem 0.13 , p. 9]). 


\section{Embedding $X^{2}$ in $F_{2}(X)$}

In this section, we study when $X^{2}$ can be embedded in $F_{2}(X)$, in the particular case when $X$ is a graph. For every $n \geq 3, T_{n}$ denotes a simple $n$-od.

Lemma 3.1. Let $n \in \mathbf{N}$, with $n \geq 3$, then $T_{n} \times T_{n}$ can not be embedded in $F_{2}\left(T_{n}\right)$.

Proof. By [5, Lemma 3.2, p. 59], $T_{n} \times T_{n}$ is homeomorphic to cone $\left(K_{n, n}\right)$. By [4, Lemma 2, p. 70], $F_{2}\left(T_{n}\right)$ is homeomorphic to cone $(Z)$, where $Z$ is the union of $K_{n}$ and $n$ pairwise disjoint arcs, each one of them intersecting $K_{n}$ in exactly one of its vertices. Suppose, to the contrary, that there exists an embedding $h: \operatorname{cone}\left(K_{n, n}\right) \rightarrow \operatorname{cone}(Z)$. Let $v$ and $v^{\prime}$ be the vertices of cone $\left(K_{n, n}\right)$ and cone $(Z)$, respectively. By $[5$, Lemma 3.3 , p. 60], each point $p \in \operatorname{cone}(Z)-$ $\left\{v^{\prime}\right\}$ has a basis of neighborhoods $\beta$ in cone $(Z)$ such that for each $U \in \beta, U$ can be embedded in $\mathbf{R}^{3}$. So, $h(v)=v^{\prime}$, which implies that the cylinder $K_{n, n} \times I$ embeds in the cylinder $Z \times I$. Looking at neighborhood bases of points of these two cylinders, we conclude that $K_{n, n}$ can be embedded in $Z$, therefore $2 n=$ $\left|R\left(K_{n, n}\right)\right| \leq|R(Z)|=n$, but this is imposible. This contradiction concludes the proof of this lemma.

The following result is obvious since $T_{m} \times[0,1]$ can be embedded in $\mathbf{R}^{3}$ while $T_{k} \times T_{l}$ can not, because cone $\left(K_{k, l}\right)$ can not be embedded in $\mathbf{R}^{3}$ (see [4, Lemma 4, p. 73]).

Lemma 3.2. Let $k, l, m \in \mathbf{N}$, with $k, l, m \geq 3$. Then $T_{k} \times T_{l}$ can not be embedded in $T_{m} \times[0,1]$.

THEOREM 3.3. If $X$ is a graph with $|R(X)| \leq 1$, then $X^{2}$ can be embedded in $F_{2}(X)$ if and only if $X$ is an arc.

Proof. If $X$ is an arc its second symmetric product is homeomorphic to $X^{2}$. Now, let $X^{2}$ embed in $F_{2}(X)$. If $|R(X)|=0, X$ is an arc or a simple closed curve (see [14, Proposition 9.5, p. 142]). Since $S^{1} \times S^{1}$ can not be embedded in $F_{2}\left(S^{1}\right)$, $X$ is an arc.

Suppose that $R(X)=\{p\}$ and that $h: X^{2} \rightarrow F_{2}(X)$ is an embedding. Let $r=\operatorname{ord}(p, X)$. So, $(p, p) \in X^{2}$ has a basis of neighborhoods $\beta$ such that if $U \in \beta$ then $U$ is homeomorphic to $T_{r} \times T_{r}$ (see [5, Lemma 3.4, p. 61]). Take 
$\{a, b\} \in F_{2}(X)$ such that $h((p, p))=\{a, b\}$. By [5, Lemma 3.3, p. 60], we have the following three cases:

Case I If $\operatorname{ord}(a, X) \leq 2$ and $\operatorname{ord}(b, X) \leq 2$, then $\{a, b\}$ has a basis of neighborhoods $\gamma$ in $F_{2}(X)$, such that if $U \in \gamma$ then $U$ is homeomorphic to $[0,1]^{2}$.

Case II If $\operatorname{ord}(a, X) \leq 2$ and $b=p$, then $\{a, b\}$ has a basis of neighborhoods $\gamma$ in $F_{2}(X)$, such that if $U \in \gamma$ then $U$ is homeomorphic to $T_{r} \times[0,1]$.

Case III If $\{a, b\}=\{p\}$, then $\{a, b\}$ has a basis of neighborhoods $\gamma$ in $F_{2}(X)$, such that if $U \in \gamma$ then $U$ is homeomorphic to $F_{2}\left(T_{r}\right)$.

We conclude that $T_{r} \times T_{r}$ can be embedded in $[0,1]^{2}, T_{r} \times[0,1]$ or $F_{2}\left(T_{r}\right)$. This is a contradiction by Lemmas 3.2 and 3.1. Then $X^{2}$ can not be embedded in $F_{2}(X)$.

THEOREM 3.4. Let $X$ be a graph. If $R(X) \neq \varnothing$ then $X^{2}$ can not be embedded in $F_{2}(X)$.

Proof. If $|R(X)|=1$ it follows by Theorem 3.3. Suppose on the contrary that $R(X)=\left\{x_{1}, \ldots, x_{k}\right\}$, with $k \geq 2$, and that there exists an embedding $h: X^{2} \rightarrow F_{2}(X)$. Let $r_{i}=\operatorname{ord}\left(x_{i}, X\right)$. It is clear that for each $i, j \in\{1, \ldots, k\}$, $\left(x_{i}, x_{j}\right) \in X^{2}$ has a basis of neighborhoods $\beta$ in $X^{2}$ such that if $U \in \beta$, then $U$ is homeomorphic to $T_{r_{i}} \times T_{r_{j}}$.

By [5, Lemma 3.3, p. 60] and Lemma 3.2, for each $i, j \in\{1, \ldots, k\}$, there exist $l, s \in\{1, \ldots, k\}$ such that $h\left(\left(x_{i}, x_{j}\right)\right)=\left\{x_{l}, x_{s}\right\}$. Therefore the sets $\left\{\left(x_{i}, x_{j}\right) \in\right.$ $\left.X^{2}: i, j \in\{1, \ldots, k\}\right\}$ and

$$
\left\{\left\{x_{i}, x_{j}\right\} \in F_{2}(X): i, j \in\{1, \ldots, k\}\right\}
$$

have the same cardinality, which is impossible.

Using the Theorems 3.3 and 3.4, we get the following result.

COROllary 3.5. Let $X$ be a graph. $X^{2}$ can be embedded in $F_{2}(X)$ if and only if $X$ is an arc.

\section{4. a-Self-Homeomorphic Continua}

Definition 4.1. Let $\alpha$ be a cardinal number and let $X$ be a continuum. We say that $X$ is $\alpha$-self-homeomorhic if there exist $\alpha$ mutually disjoint proper subcontinua of $X$ which are homeomorphic to $X$. 
REMARK 4.2. It is clear that every $n$-cell is $m$-self-homeomorphic for each $m, n \in \mathbf{N}$.

Proposition 4.3. Let $n \in \mathbf{N}$ and $X$ be a continuum. If $X$ is n-selfhomeomorphic then $X^{m}$ can be embedded in $F_{m}(X)$ for each $m \leq n$.

Proof. Let $m \leq n$. Since $X$ is $n$-self-homeomorphic, $X$ is $m$-selfhomeomorphic. Therefore, there exist pairwise disjoint subcontinua $A_{1}, \ldots, A_{m}$ of $X$, such that $A_{i}$ is homeomorphic to $X$. Consider the map $f_{m}^{X}: X^{m} \rightarrow F_{m}(X)$ given by $f_{m}^{X}\left(\left(x_{1}, \ldots, x_{m}\right)\right)=\left\{\underset{m}{\left.x_{1}, \ldots, x_{m}\right\}}\right.$ (see [2, p. 877]). So, $\left.f_{m}^{X}\right|_{\prod_{i=1}^{m} A_{i}}: \prod_{i=1}^{m} A_{i} \rightarrow$ $F_{m}(X)$ is an embedding. Since $\prod_{i=1}^{m} A_{i}$ is homeomorphic to $X^{m}$, we conclude that $X^{m}$ can be embedded in $F_{m}(X)$.

Proposition 4.4. Let $X$ be a continuum. $X$ is 2-self-homeomorphic if and only if $X$ is $n$-self-homeomorphic for each $n \geq 2$.

Proof. It follows from Definition 4.1.

As a consequence of propositions 4.3 and 4.4 we have the following result.

Corollary 4.5. Let $X$ be a continuum. If $X$ is 2-self-homeomorphic then $X^{n}$ can be embedded in $F_{n}(X)$ for each $n \in \mathbf{N}$.

COROllary 4.6. Let $X$ be a graph. $X$ is 2-self-homeomorphic if and only if $X$ is an arc.

Proof. It follows from Corollary 3.5 and Proposition 4.4.

Corollary 4.7. Let $X$ be a continuum. If $X$ contains a Hilbert cube, then $X^{n}$ can be embedded in $F_{n}(X)$ for each $n \in \mathbf{N}$.

PRoOF. It follows from Corollary 4.5 and [12, Theorem 1, p. 241].

The following definitions appear in [7, p. 217] and [6, p. 283-284].

Definition 4.8. A topological space $X$ is self-homeomorphic (strongly selfhomeomorphic, respectively) if for any open set $U \subset X$ there is a set $V \subset U$ (with nonempty interior, respectively) homeomorphic to $X$. 
Definition 4.9. A topological space $X$ is pointwise self-homeomorphic (strongly pointwise self-homeomorphic, respectively) at a point $x \in X$ if for any neighborhood $U$ of $x$ there is a set $V$ (a neighborhood of $x$, respectively) such that $x \in V \subset U$ and $V$ is homeomorphic to $X$. A space $X$ is pointwise selfhomeomorphic (strongly pointwise self-homeomorphic, respectively) if it is pointwise self-homeomorphic (strongly pointwise self-homeomorphic, respectively) at each point.

The following fact is easy to see, compare with [7, Theorem 2.5, p. 217].

Proposition 4.10. Definitions $4.1,4.8$ and 4.9 satisfy: $4.1 \Rightarrow 4.8 \Rightarrow 4.9$.

Concerning this proposition, in [7, Problems 6.21 and 6.23, p. 237] the authors asked whether $X$ is pointwise self-homeomorphic if $X$ is a selfhomeomorphic or a strongly self-homeomorphic dendrite. A negative answer to both these questions is given in [15], where a dendrite is constructed which is strongly self-homeomorphic (at each of its points) but not pointwise selfhomeomorphic (at some of its end points). In [8], the authors generalize the Pyrih's example given in [15].

COROLLARY 4.11. There are uncontably many topologically different dendrites such that $X^{n}$ can be embedded in $F_{n}(X)$ for each $n \in \mathbf{N}$.

Proof. This is a consequence of Propositions 4.3, 4.10 and [7, Corollary 6.5 , p. 230].

The following proposition on cartesian products is easy to see, compare with [7, Proposition 2.11, p. 219].

Proposition 4.12. All types of self-homeomorphic spaces considered above are preserved by Cartesian products (of arbitrarily many factors).

Other examples of continua such that $X^{n}$ can be embedded in $F_{n}(X)$ are the Sierpiński universal curve, the Sierpiński triangle, the Menger curve, the familly of plane continua constructed in [6, Section 4] and by the previus proposition, cartesian products with factors being $\alpha$-self-homeomorphic continua. 
EXAMPLE 4.13. There exists a continuum $X$ such that for each $m, n \in \mathbf{N}, X^{n}$ can be embedded in $F_{n}(X)$ but $X$ is not $m$-self-homeomorphic.

Proof. Consider $Y$ a continuum homeomorphic to the capital letter $H$. Let $X=Y \times I$ and $A_{1}, A_{2}, \ldots, A_{n}$ be pairwise disjoint cylinders in $X$, each one of them homeomorphic to $X$. Then $\left\langle A_{1}, \ldots, A_{n}\right\rangle$ is homeomorphic to $X^{n}$ and is contained in $F_{n}(X)$, but $X$ is not $m$-self-homeomorphic for every $m \in \mathbf{N}$.

Question 4.14. Does there exist a dendrite $X$ such that $X^{n}$ can be embedded in $F_{n}(X)$ and $X$ is not m-self-homeomorphic, for $m, n \in \mathbf{N}$ ?

QUESTION 4.15. Does there exist a continuum $X$ such that $X^{n}$ can be embedded in $F_{n}(X)$ but $X$ does not contain a proper subcontinuum $Y$ homeomorphic to $X$ ?

\section{Acknowledgment}

The authors thank the referee's suggestion, which helped to substantially improve the writing of this paper.

\section{References}

[1] K. Borsuk, On the third symmetric potency on the circumference, Fund. Math. 36 (1949), 235-244.

[2] K. Borsuk and S. Ulam, On symmetric products of topological spaces, Bull. Amer. Math. Soc. 37 (1931), 875-882.

[ 3 ] R. Bott, On the third symmetric potency of $S_{1}$, Fund. Math. 39 (1952), 364-368.

[4] E. Castañeda, Embedding symmetric products in euclidean spaces, Continumm Theory. Lecture Notes in Pure Appl. Math. 230. New York: Dekker, (2002), 67-79.

[ 5 ] E. Castañeda, Symmetric products as cones and products, Topology Proc. 28 (2004), 55-67.

[6] W. J. Charatonik, A. Dilks Dye and J. Read, Self-homeomorphic star figures, Continuum Theory and Dynamical Systems, Thelma West (Editor), Lecture Notes in Pure and Applied Mathematics, 149, Marcel Dekker, (1993), 283-290.

[7] W. J. Charatonik and A. Dilks, On self-homeomorphic spaces, Topology Appl. 55 (1994), 215-238.

[8] J. J. Charatonik and P. Krupski, On self-homeomorphic dendrites, Comment. Math. Univ. Carolinae 43 (2002), 665-673.

[9] J. Dugundji, Topology, Allyn and Bacon, Inc., Boston, 1966.

[10] A. Illanes, Hiperespacios de Continuos (Spanish), Aportaciones Matemáticas 28, Sociedad Matemática Mexicana, México, (2004).

[11] R. Molski, On symmetric products, Fund. Math. 44 (1957), 165-170.

[12] K. Kuratowski, Topology, Vol. I, Academic Press, New York, N. Y., 1966.

[13] S. B. Nadler, Jr., Hyperspaces of Sets, Monographs and Textbooks in Pure and Applied Mathematics, 49, New York: Marcel Dekker, Inc., 1978. 
[14] S. B. Nadler, Jr., Continuum Theory an Introduction, Monographs and Textbooks in Pure and Appied Mathematics, 158, New York: Marcel Dekker, Inc., 1992.

[15] P. Pyrih, An example of strongly self-homeomorphic dendrite not pointwise self-homeomorphic, Comment. Math. Univ. Carolinae, 40 (1999), 571-576.

Enrique Castañeda-Alvarado

Universidad Autónoma del Estado de México

Facultad de Ciencias, Instituto Literario No. 100

Col. Centro, C.P. 50000, Toluca

Estado de México, México

E-mail: eca@uaemex.mx

Javier Sánchez-Martínez

Benemérita Universidad Autónoma de Puebla

Facultad de Ciencias Físico-Matemáticas

Av. San Claudio y 18 Sur, Edificio 11A-109B

Col. San Manuel, Ciudad Universitaria

C.P. 72570, Puebla, Puebla

E-mail: jsanchezm@fcfm.buap.mx 